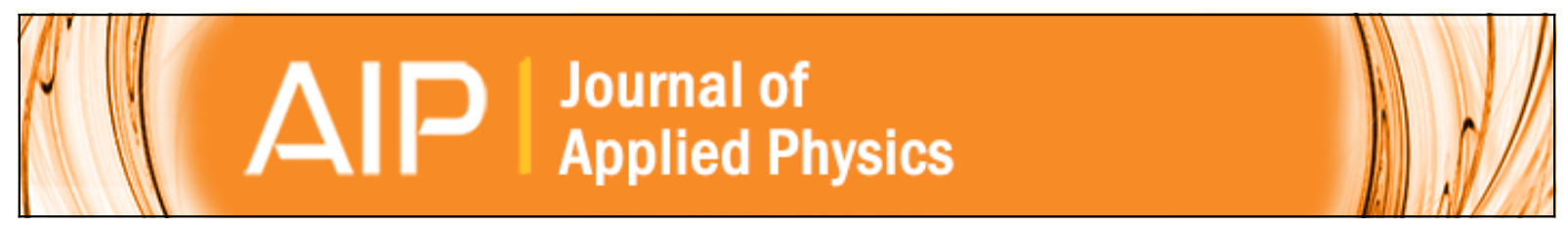

\title{
Electron acceleration by a short laser beam in the presence of a long-wavelength electromagnetic wave
}

Devki Nanadan Gupta, Sandeep Kumar, Moohyun Yoon, Min Sup Hur, and Hyyong Suk

Citation: Journal of Applied Physics 102, 056106 (2007); doi: 10.1063/1.2778286

View online: http://dx.doi.org/10.1063/1.2778286

View Table of Contents: http://scitation.aip.org/content/aip/journal/jap/102/5?ver=pdfcov

Published by the AIP Publishing

\section{Articles you may be interested in}

Comment on "Electron acceleration by a short laser beam in the presence of a long-wavelength electromagnetic wave" [J. Appl. Phys.102, 056106 (2007)]

J. Appl. Phys. 111, 106102 (2012); 10.1063/1.4719080

Laser plasma acceleration of electrons: Towards the production of monoenergetic beamsa)

Phys. Plasmas 12, 056711 (2005); 10.1063/1.1902951

Observation of mono-energetic structures in the spectrum of laser wakefield accelerated electrons AIP Conf. Proc. 737, 853 (2004); 10.1063/1.1842633

Propagation In Matter Of Currents Of Relativistic Electrons Beyond The Alfven Limit, Produced In Ultra-HighIntensity Short-Pulse Laser-Matter Interactions

AIP Conf. Proc. 740, 446 (2004); 10.1063/1.1843526

On the beam cross section of a high-gain Compton free-electron laser

Phys. Plasmas 9, 1471 (2002); 10.1063/1.1460884

\section{MIT LINCOLN} LABORATORY CAREERS

Discover the satisfaction of innovation and service to the nation
- Space Control

- Air \& Missile Defense

- Communications Systems \& Cyber Security

- Intelligence, Surveillance and

Reconnaissance Systems

$$
\begin{aligned}
& \text { - Advanced } \\
& \text { Electronics } \\
& \text { - Tactical Systems } \\
& \text { - Homeland } \\
& \text { Protection } \\
& \text { - Air Traffic Control }
\end{aligned}
$$

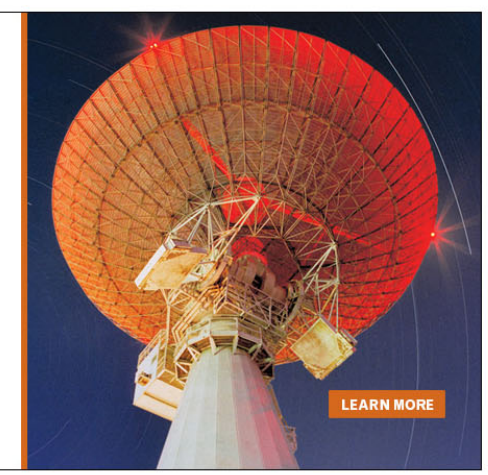




\title{
Electron acceleration by a short laser beam in the presence of a long-wavelength electromagnetic wave
}

\author{
Devki Nanadan Gupta ${ }^{\text {a) }}$ \\ Center for Advanced Accelerators, Korea Electrotechnology Research Institute (KERI), Changwon 641-120, \\ Korea

\begin{abstract}
Sandeep Kumar and Moohyun Yoon
Department of Physics and Center for Theoretical Physics, Pohang University of Science and Technology (POSTECH), Pohang 790-784, Korea

Min Sup Hur

Center for Advanced Accelerators, Korea Electrotechnology Research Institute (KERI), Changwon 641-120, Korea

Hyyong Suk ${ }^{\text {b) }}$

Advanced Photonics Research Institute, Gwangju Institute of Science and Technology (GIST), Gwangju 500-712, Korea
\end{abstract}

(Received 25 June 2007; accepted 24 July 2007; published online 13 September 2007)

\begin{abstract}
A scheme for laser-induced acceleration of an electron injected initially at an angle to the direction of a short-wavelength laser is investigated, where an additional long-wavelength electromagnetic wave is introduced to achieve high energy gain. Due to the beating effect of the electromagnetic waves, the electron can gain additional energy. Some computational results are presented to estimate the electron energy gain by the proposed scheme, where the gain increases by increasing the difference of the wavelengths. (C) 2007 American Institute of Physics. [DOI: 10.1063/1.2778286]
\end{abstract}

Development of ultrahigh power, short pulse lasers based on chirped-pulse amplification technique has been intensively pursued for many years. ${ }^{1}$ Consequently, there have emerged many new frontier research areas. Among them, the laser particle accelerations in vacuum and plasma are very important. ${ }^{2-5}$ However, there are some fundamental limitations in vacuum electron accelerations. One of them is that the electric field distribution is extremely complex in the focal plane of a linearly polarized, focused Gaussian beam. The significant transverse field components tend to deflect the particles and increase the beam emittance. Second, the interaction length is limited so that the oscillatory electromagnetic field does not cancel out any net acceleration. Vacuum as a medium for electron acceleration has some advantages over plasma. In vacuum the problems inherent in laser-plasma interaction, ${ }^{6}$ such as instabilities, are absent. Furthermore, the peak energy attained by the electron increases with the initial electron energy and it is easier to inject preaccelerated electrons in vacuum than in a plasma. $^{7-9}$

Several mechanisms are proposed to laser accelerations of relativistic electrons for minimize the accelerator cost. ${ }^{10-12}$ Esarey et al..$^{13}$ have discussed several theoretical features of vacuum. The experimental results on the $\mathrm{MeV}$ electron generation by the Lorentz force of an ultraintense linearly polarized laser pulse in vacuum were reported by Malka et al. ${ }^{14}$ As previously reported in our work, ${ }^{15,16}$ the most surprising and meaningful result for vacuum electron acceleration in the presence of a magnetic field is that, when a suitable frequency chirp of the laser is introduced, the electron not only

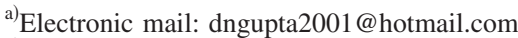

${ }^{b)}$ Electronic mail: hyyongsuk@yahoo.co.kr
}

accelerates to high energy but also retains it even after passing of the laser pulse. Recently, Kawata et al. ${ }^{17}$ have studied the electron ponderomotive acceleration in vacuum by an intense short-pulsed laser, where the electron trapping and further electron acceleration was discussed. A good quality electron beam was generated by this mechanism.

The electron energy gain during laser acceleration in vacuum mainly depends on the laser intensity, because the longitudinal momentum of the electron is proportional to the square of the laser intensity amplitude. In order to achieve the desired high-energy gains in vacuum, therefore, a study of electron dynamics needs to be conducted in the higher laser fields. The required fields can be realized by using two electromagnetic waves. In this communication, we propose an electron acceleration mechanism by a short-wavelength electromagnetic wave in the presence of a long-wavelength electromagnetic wave. An electron can be trapped by the long electromagnetic wave and it can stay in the interaction region longer. A short electromagnetic wave may further accelerate the trapped electron to higher energy. We consider a plane-polarized short-wavelength laser with electric fields (propagating in the positive $z$ direction)

$$
\begin{aligned}
E_{x}= & \frac{A_{0}}{f} \cos (\phi) \exp \left[-\frac{r^{2}}{r_{0}^{2} f^{2}}\right], \\
E_{z}= & -\frac{A_{0}}{f}\left[\frac{2 x}{k_{0} r_{0}^{2} f^{2}} \sin (\phi)+\frac{x}{z\left[1+\left(R_{d} / z\right)^{2}\right]} \cos (\phi)\right] \\
& \times \exp \left[-\frac{r^{2}}{r_{0}^{2} f^{2}}\right],
\end{aligned}
$$

where $\phi=\omega_{0} t-k_{0} z+\tan ^{-1}\left(z / R_{d}\right)-k_{0} r^{2} / 2 z\left[1+\left(R_{d} / z\right)^{2}\right], f^{2}=1$ $+\left(z / R_{d}\right)^{2}, k_{0}=\omega_{0} / c, A_{0}$ is the amplitude of the field, $R_{d}$ 


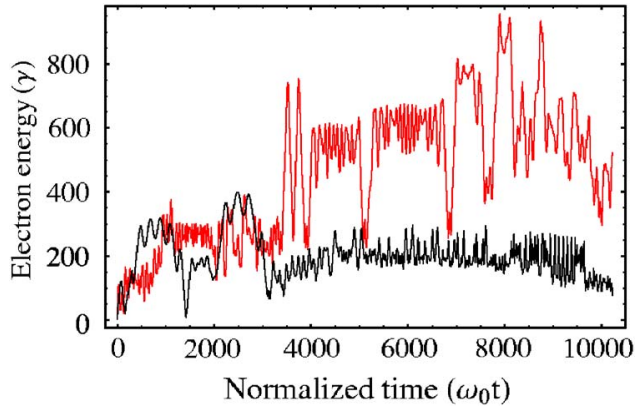

FIG. 1. (Color online) Variation of the electron energy $(\gamma)$ with time (normalized by $\left.1 / \omega_{0}\right)$ for different initial electron energies $\gamma_{0}=1.5$ (black curve) and $\gamma_{0}=5$ (red curve). The used lasers parameters are $a_{0}=1, r_{0}=25 \mu \mathrm{m}$, $\lambda_{0}=1 \mu \mathrm{m}, a_{1}=0.4, \lambda_{1}=10 \mu \mathrm{m}$, and $r_{1}=40 \mu \mathrm{m}$. An electron of finite initial energy is injected at an angle $\theta=10^{\circ}$.

$=k_{0} r_{0}^{2} / 2$ is the Rayleigh length, $r^{2}=x^{2}+y^{2}+z^{2}, r_{0}$ is the minimum laser spot size, and $c$ is the velocity of light in vacuum. The magnetic field related to the laser pulse is given by $\nabla$ $\times \mathbf{E}=-\partial \mathbf{B} / \partial t$. We also consider another long-wavelength electromagnetic wave, which propagates in the negative $z$ direction. The fields $\left(\mathbf{E}_{1}, \mathbf{B}_{1}\right)$ of the long electromagnetic wave can be written from Eqs. (1) and (2) by replacing the subscript 0 to 1 and by choosing the opposite propagation direction of the wave vector.

An electron is injected externally at a certain angle $\theta$ to the $z$ direction. The equations governing the electron momentum and energy can be written as

$$
\begin{aligned}
& \frac{d \mathbf{p}}{d t}=-e\left(\mathbf{E}^{\prime}+\frac{1}{c} \boldsymbol{v} \times \mathbf{B}^{\prime}\right), \\
& \frac{d \gamma}{d t}=-\frac{e}{m c^{2}} \mathbf{E}^{\prime} \cdot \boldsymbol{v},
\end{aligned}
$$

where $\mathbf{E}^{\prime}=\mathbf{E}+\mathbf{E}_{1}, \mathbf{B}^{\prime}=\mathbf{B}+\mathbf{B}_{1}, \mathbf{p}=\gamma m \boldsymbol{v}, \quad \gamma=\left(1-v^{2} / c^{2}\right)^{-1 / 2}$ is the relativistic factor and $-e$ and $m$ are the electron's charge and rest mass, respectively.

We can solve Eqs. (3) and (4) numerically for electron energy. We assume that the electron is injected initially at an angle $\theta$ to the direction of propagation of the short wavelength laser pulse with $\mathbf{p}_{0}=\hat{x} p_{x 0} \sin \theta+\hat{z} p_{z 0} \cos \theta$, where $p_{0}$ $=\gamma_{0} m_{0} v_{0}$ is the initial momentum of the electron, $v_{0}$ is the initial velocity of the electron, and $\gamma_{0}$ is the initial kinetic energy of the electron. In our calculations, time and length are normalized by $1 / \omega_{0}$ and $c / \omega_{0}$, and momentum and energy are normalized by $m c$ and $m c^{2}$, respectively. The parameters of the short pulse laser wave are chosen for a Ti:sapphire Nd:glass laser of intensity $I_{0}=1.37 \times 10^{18} \mathrm{~W} / \mathrm{cm}^{2}\left(a_{0}\right.$ $\left.=e A_{0} / m_{0} \omega_{0} c=1\right)$. The laser spot size and wavelength are $r_{0}$ $=25 \mu \mathrm{m}$ and $\lambda_{0}=1 \mu \mathrm{m}$, respectively. We choose the counter propagating long-wavelength electromagnetic wave of intensity $I_{0}=2.2 \times 10^{15} \mathrm{~W} / \mathrm{cm}^{2}\left(a_{1}=e A_{1} / m_{0} \omega_{1} c=0.4\right.$, corresponding to the wavelength $\lambda_{1}=10 \mu \mathrm{m}$ and spot size $r_{1}=40 \mu \mathrm{m}$ ). An electron of finite initial energy is injected at an angle $\theta$ $=10^{\circ}$.

We examine the given parameters to solve the coupled differential equations [Eqs. (3) and (4)]. Figure 1 shows the electron energy $(\gamma)$ with time (normalized by $\left.1 / \omega_{0}\right)$ for different initial electron kinetic energies $\gamma_{0}=1.5$ (black curve)

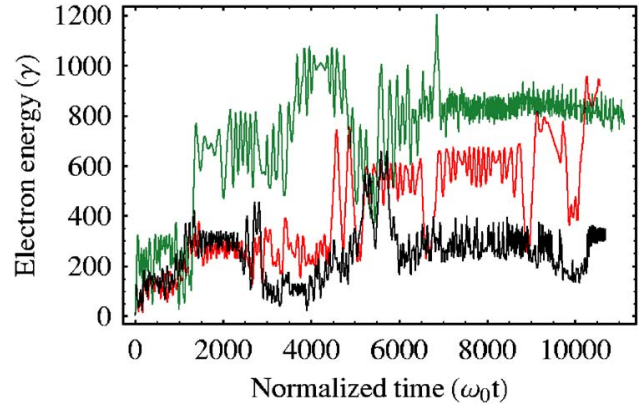

FIG. 2. (Color online) Variation of the electron energy $(\gamma)$ with time (normalized by $\left.1 / \omega_{0}\right)$ for different wavelengths of the long counterpropagating electromagnetic wave $\lambda_{1} \approx 8 \mu \mathrm{m}$ (black curve), $\lambda_{1} \approx 10 \mu \mathrm{m}$ (red curve), and $\lambda_{1} \approx 15 \mu \mathrm{m}$ (green curve). The used laser parameters are $a_{0}=1, r_{0}=25 \mu \mathrm{m}$, and $\lambda_{0}=1 \mu \mathrm{m}$. Other parameters of the counterpropagating electromagnetic wave are used corresponding to the given wavelengths. An electron of finite initial energy $\left(\gamma_{0}=5\right)$ is injected at an angle $\theta=10^{\circ}$.

and $\gamma_{0}=5$ (red curve). Our result indicates that the electron experiences a ponderomotive force due to the combined fields of the both electromagnetic waves. Hence, the electron is trapped by the long-generated beating of two counterpropagating electromagnetic waves of greatly different wavelengths, keeping the electron in the accelerating phase over the full interaction region. Therefore, the electron acquires more energy from the interacting electromagnetic fields. Here, it should be noted that the energy gain of the electron during acceleration is sensitive to the initial electron energy. Our study shows that enhancement of the electron energy was observed for high initial electron kinetic energy. In other words, the electron can gain high energy if it is preaccelerated, because the duration of the interaction between the interacting waves and the electron increases with the initial electron energy. Our equations yield about $450 \mathrm{MeV}$ electron energy gain if an electron of initial kinetic energy of $2.5 \mathrm{MeV}$ is injected into the interaction region. Figure 2 shows the time dependent variation of the electron energy for different wavelengths of the counterpropagating electromagnetic waves. The used wavelengths are $\lambda_{1}$ $\approx 8 \mu \mathrm{m}$ (black curve), $\lambda_{1} \approx 10 \mu \mathrm{m}$ (red curve), and $\lambda_{1}$ $\approx 15 \mu \mathrm{m}$ (green curve). The intensity amplitudes of couterpropagating electromagnetic waves are used corresponding to the given wavelengths. It is observed that the electron energy gain increases as the difference of the wavelengths of both electromagnetic waves $\left(\lambda_{1}-\lambda_{0}\right)$ is increased. The longer wavelength of the counterpropagating electromagnetic wave increases the acceleration phase region, which may allow the electron to stay in the acceleration region longer. Hence, in the proposed mechanism, an electron can get more energy if a longer-wavelength electromagnetic wave is present.

In conclusion, we proposed a scheme for vacuum acceleration of an externally injected electron by a shortwavelength electromagnetic wave in the presence of a longwavelength electromagnetic wave. Beating of both waves leads to trapping and acceleration of an electron, so that the electron can be accelerated to a considerably higher energy. The enhancement in electron energy is due to the fact that the ponderomotive force at the beat frequency (generated by the two electromagnetic waves of different wavelengths) 
keeps the electron in the accelerating phase over a full interaction region. In this mechanism, the acquired energy can increase if the injected electron energy is higher. Furthermore, the increasing wavelength of the counterpropagating wave can make this mechanism more efficient. The main advantage of this mechanism is that one may obtain a considerably high electron-beam energy by using two lowintensity laser beams. By today's technology, this mechanism may be realized easily.

This work was supported by the Korean Ministry of Science and Technology through the Creative Research Initiative Program/KOSEF and Ministry of Education and Human Resources, Korea.

${ }^{1}$ M. D. Perry and G. A. Mourou, Science 264, 917 (1994).

${ }^{2}$ H. Hora, Nature (London) 333, 337 (1988); T. Hauser, W. Scheid, and H. Hora, Phys. Lett. A 186, 189 (1994); J. X. Wang, Y. K. Ho, L. Feng, Q. Kong, P. X. Wang, Z. S. Yuan, and W. Scheid, Phys. Rev. E 60, 7473 (1999).

${ }^{3}$ D. N. Gupta and C. M. Ryu, Phys. Plasmas 12, 053103 (2005); D. N. Gupta and H. Suk, Laser Part. Beams 25, 31 (2007).

${ }^{4}$ J. Badziak, S. Glowacz, S. Jablonski, P. Parys, J. Wolowski, and H. Hora, Appl. Phys. Lett. 85, 3041 (2004); L. Yin, B. J. Albright, B. M. Hegelich, and J. C. Fernandez, Laser Part. Beams 24, 291 (2006); Y. Glinec, J. Faure, A. Pukhov, S. Kiselev, S. Gordienko, B. Mercier, and V. Malka, ibid. 23, 161 (2005).

${ }^{5}$ R. Bingham, J. T. Mendonca, and P. K. Shukla, Plasma Phys. Controlled Fusion 46, R1 (2004); D. Umstadter, Phys. Plasmas 8, 1774 (2001); B. Hafizi, A. Ting, E. Esarey, P. Sprangle, and J. Krall, Phys. Rev. E 55, 5924 (1997); D. N. Gupta and H. Suk, J. Appl. Phys. 101, 114908 (2007).

${ }^{6}$ K. Sakai, S. Miyazaki, S. Kawata, S. Hasumi, and T. Kikuchi, Laser Part. Beams 24, 321 (2006); M. Roth et al., ibid. 23, 95 (2005).

${ }^{7}$ F. V. Hartemann, J. R. Van Meter, A. L. Troha, E. C. Landahl, N. C. Luhmann, Jr., H. A. Baldis, A. Gupta, and A. K. Kerman, Phys. Rev. E 58, 5001 (1998); E. Esarey, P. Sprangle, J. Krall, and A. Ting, IEEE Trans. Plasma Sci. 24, 252 (1996).

${ }^{8} \mathrm{D}$. Li and K. Imasaki, Appl. Phys. Lett. 87, 091106 (2005); T. Plettner, R. L. Byer, E. Colby, B. Cowan, C. M. S. Sears, J. E. Spencer, and R. H. Siemann, Phys. Rev. Lett. 95, 134801 (2005); Y. Cheng and Z. Xu, Appl. Phys. Lett. 74, 2116 (1999).

${ }^{9}$ Y. I. Salamin, Phys. Rev. A 73, 043402 (2006); Y. I. Salamin and C. H. Keitel, Appl. Phys. Lett. 77, 1082 (2000).

${ }^{10}$ B. Rau, T. Tajima, and H. Hojo, Phys. Rev. Lett. 78, 3310 (1997).

${ }^{11}$ T. Nakamura, H. Sakagami, T. Johzaki, H. Nagamoto, and K. Mima, Laser Part. Beams 24, 5 (2006); A. F. Lifshitz, J. Faure, Y. Glinec, V. Malka, and P. Mora, ibid. 24, 255 (2006); K. Koyama, M. Adachi, E. Miura, S. Kato, S. Masuda, T. Watanabe, A. Ogata, and M. Tanimoto, ibid. 24, 95 (2006). ${ }^{12}$ P. X. Wang, W. Scheid, and Y. K. Ho, Appl. Phys. Lett. 90, 111113 (2007).

${ }^{13}$ E. Esarey, P. Sprangle, and J. Krall, Phys. Rev. E 52, 5443 (1995).

${ }^{14}$ G. Malka, E. Lefebvre, and J. L. Miquel, Phys. Rev. Lett. 78, 3314 (1997).

${ }^{15}$ D. N. Gupta and H. Suk, Phys. Plasmas 13, 044507 (2006); 13, 013105 (2006).

${ }^{16}$ D. N. Gupta, M. S. Hur, and H. Suk, Phys. Plasmas 14, 044701 (2007).

${ }^{17}$ S. Kawata et al., Laser Part. Beams 23, 61 (2005). 